Rev. High Pressure Sci. Technol., Vol. 7 (1998) 623 625

\title{
Anomalous Compressibility Associated with First Order Valence Change in $\mathrm{YbInCu}_{4}$
}

\author{
Z. Arnold, J. Kamarád, J.M. De Teresa*, and M.R.Ibarra* \\ Institute of Physics, Academy of Sciences of Czech Republic, Cukrovarnická 10, 16200 Prague 6, Czech Republic \\ *Dpto Física de la Materia Condensada-ICMA, Facultad de Ciencias, Universidad de Zaragoza-CSIC, 50009 Zaragoza, Spain
}

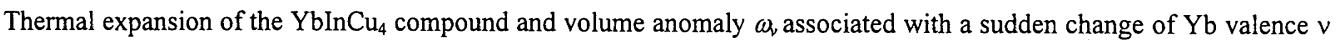
at $T_{V}$ were measured under pressure up to $10 \mathrm{kbar}$. Both, the temperature $T_{V} \approx 40 \mathrm{~K}$ and the anomaly $\omega_{v}=0.43 \%$ decrease under pressure linearly (with slopes $d T_{V} / d p=-2.2 \mathrm{~K} / \mathrm{kbar}^{-1}$ and $d \omega, / d p=-0.021 \% \mathrm{kbar}^{-1}$ ) up to a critical pressure $p_{C} \cong 20 \mathrm{kbar}$. The compressibility $\kappa$ increases below $T_{V}, \kappa=1.22 \mathrm{Mbar}^{-1}$ at $20 \mathrm{~K}$ and $\kappa=0.99 \mathrm{Mbar}^{-1}$ at 295 $\mathrm{K}$. A derived pressure change of $v$ (or $f$-shell occupation $n_{f}$ ), $d v / d p \approx 0.005 \mathrm{kbar}^{-1}$, leads to $d \ln V_{Y b} / d n_{f} \cong 0.15$. [valence fluctuation, thermal expansion, compressibility, rare earth intermetallics]
\end{abstract}

\section{Introduction}

During the last years, a great attention has been paid to the study of the $\mathrm{YbInCu}_{4}$ compound that belongs to a family of mixed-valence systems. Very sudden and pronounced changes of its physical properties, as a sharp volume increase and an abrupt decrease of electrical resistivity and magnetic susceptibility, have been observed at temperature $T_{V} \approx 40 \mathrm{~K}$. At the same time, the $\mathrm{C} 15 \mathrm{~b}$ cubic crystal structure of the $\mathrm{YbInCu}_{4}$ compound remains stable, unaffected by these significant changes[1-4]. The sudden change of the $\mathrm{Yb}$ valence $v$ (from $\mathrm{Yb}^{+3}$ above $T_{V}$ to an intermediate valence $\mathrm{Yb}^{+2.9}$ below $T_{V}$ ) was deduced from the above mentioned measurement, $\mathrm{X}$-ray absorption spectra and $\mathrm{Cu}$ nuclear quadrupole resonance study at low temperatures [1-5]. It was accepted that the $4 f$ electrons of $\mathrm{Yb}$ become suddenly hybridized with conduction $d$ - electrons in $\mathrm{YbInCu}_{4}$ below $T_{V}$. The first-order-like $\mathrm{Yb}$ valence change in $\mathrm{YbInCu}_{4}$ differs in this sense from the known continuous valence transition in pure $\mathrm{Yb}$ under pressure [6].

The first-order valence change is accompanied by an anomalous volume expansion with decreasing temperature below $T_{V}$. This has been a reason for very intensive pressure study of these phenomena in the last years $[2,7-10]$. In this contribution we present the results of the study of the effect of pressure on the volume anomaly in the $\mathrm{YbInCu}_{4}$ compound based on compressibility and thermal expansion measurements under pressure.

\section{Experimental details and Results}

The polycrystalline $\mathrm{YbInCu}_{4}$ compound was prepared by arc melting of the constituent elements. The phase purity was checked by X-ray powder diffraction technique at room temperature. A SQUID magnetometer was used to measure the ac-susceptibility of $\mathrm{YbInCu}_{4}$ in the temperature range $5-300 \mathrm{~K}$ and to determine the temperature $T_{\nu}$. The real part of the susceptibility $\chi^{\prime}$ drops abruptly at $T_{V} \approx 40 \mathrm{~K}$ reflecting the transition to the mixed-valence state. The imaginary part of the susceptibility $\chi$ " shows a rapid increase below $T_{V}$. It can be related to the noticed decrease of the resistivity and relevant increase of eddy currents in the sample. The temperature dependence of $1 / \chi^{\prime}$ is plotted on Fig. 1. The linear fit of the curve above $T_{V}$ follows the simple Curie law with Weiss constant $\theta=0$ and $\mu_{\text {eff }}=4.41 \mu_{\mathrm{B}}$, very close to the free $\mathrm{Yb}^{+3}$ ion magnetic moment $\left(4.54 \mu_{\mathrm{B}}\right)$. This behaviour of ac-susceptibility verifies the good quality of the studied samples.

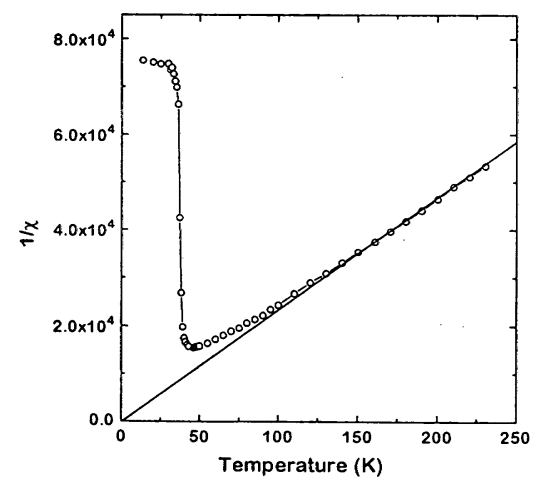

Fig. 1. Dependence of $1 / \chi^{\prime} v s$. $T$ under normal pressure

The compressibility and the thermal expansion of $\mathrm{YbInCu}_{4}$ were measured by micro-strain gauges (MicroMeasurements Inc., SK-350) in temperature range from $10 \mathrm{~K}$ to $300 \mathrm{~K}$. The strain gauges were calibrated using the relevant reference data of compressibility and thermal expansion of silica, $\mathrm{Cu}$ and $\mathrm{Fe}$. The pressure experiments were performed in a $\mathrm{CuBe}$ cell with fixed 
hydrostatic pressure up to $9 \mathrm{kbar}$. The pressure was measured in situ using a manganin pressure sensor.

The linear thermal expansions of the $\mathrm{YbInCu}_{4}$ and the isostructural $\mathrm{LuInCu}_{4}$ compounds are compared in Fig.2 to determine the volume anomaly at $T_{V}$ and its pressure dependence. (To eliminate the pressure effect on the $\mathrm{Yb}^{+3}$ phase above $T_{V}$, the curves were shifted to coincide with the $\mathrm{LuInCu}_{4}$ data at $\mathrm{T}>100 \mathrm{~K}$.) The increase of volume due to the first-order $\mathrm{Yb}$ valence change in the $\mathrm{YbInCu}_{4}$ compound is $\omega_{v}=3 \Delta L / L=0.43 \%$ under normal pressure (it well agrees with $0.45 \%$, determined by X-ray diffraction $[1,4])$ and it decreases with a slope of $d \omega_{v} / d p=-0.021 \% \mathrm{kbar}^{-1}$ under increasing pressure.

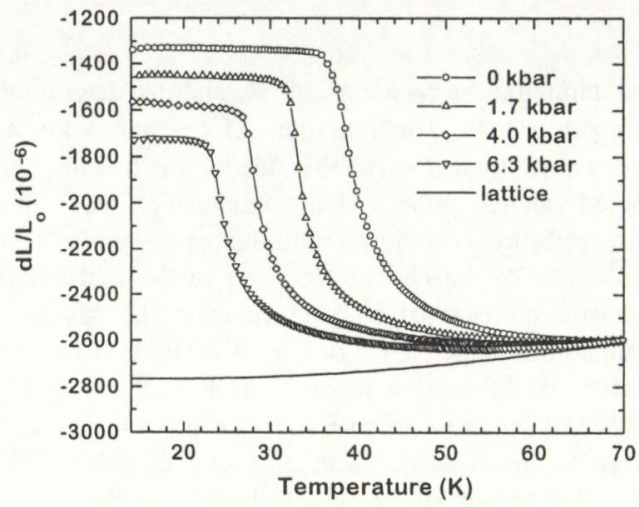

Fig. 2. Linear thermal expansion of $\mathrm{YbInCu}_{4}$ vs. temperature under several pressures. A dotted "lattice" curve represents the thermal expansion of $\mathrm{LuInCu}_{4}$.

$T_{V}$ was determined as the temperature of the maximum on the thermal expansion coefficient curve under pressure (see Fig. 3). A linear fit of the pressure shift of $T_{V}$ gives the value :

$$
d T_{V} / d p=-2.2 \mathrm{Kkbar}^{-1}
$$

which is in a good agreement with previous pressure results $[2,7-10]$.

Both parameters $\left(d \ln \omega_{\nu} / d p\right)^{-1}$ and $\left(d \ln T_{V} / d p\right)^{-1}$ give the same value of the critical pressure $p_{C} \cong 20 \mathrm{kbar}$ for a loss of the valence change phenomenon. Using this value of $p_{C}$ and the relevant increase of the $\mathrm{Yb}$ valence in the $\mathrm{YbInCu}_{4}$ compound, $\Delta v=0.1$, we can estimate the pressure rate of the $\mathrm{Yb}$ valence change as :

$$
d v / d p \approx 0.005 \text { kbar }^{-1}
$$

This is in good agreement with the previous estimation $\left(d v / d p \approx 0.0045 \mathrm{kbar}^{-l}\right)$ in [10], based on an interpolation of thermal expansion of the $\mathrm{YbAl}_{2}, \mathrm{TmAl}_{2}$ and $\mathrm{LuAl}_{2}$ compounds.
The compressibility has been measured either directly at room temperature or calculated from the linear thermal

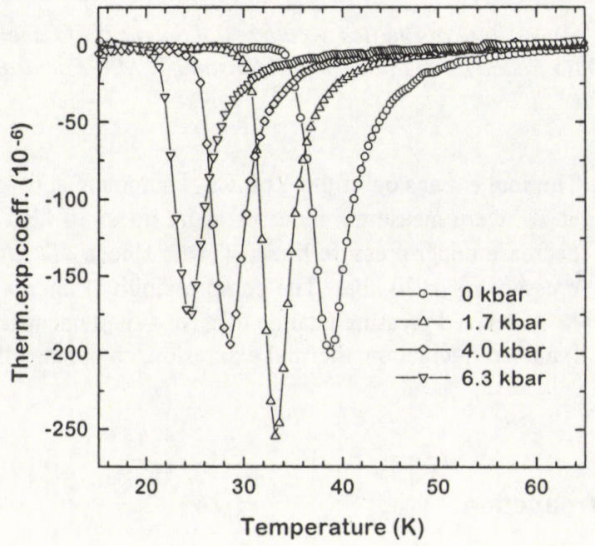

Fig. 3. Temperature and pressure dependence of the linear thermal expansion coefficient $\alpha$ of the $\mathrm{YbInCu}_{4}$ compound

expansion measurements under fixed pressures. In the latter case, an intrinsic temperature and pressure response of the micro-strain gauges and a decrease of pressure in the cooled pressure cell were taken into account. Values of the isothermal compressibility $\kappa=$ $(1 / V)(d V / d p)=-3(d L / L) / d p$ has been obtained as a linear fit of $V(p) / V(0)$ vs. $p$ data. The results are presented in Fig. 4.

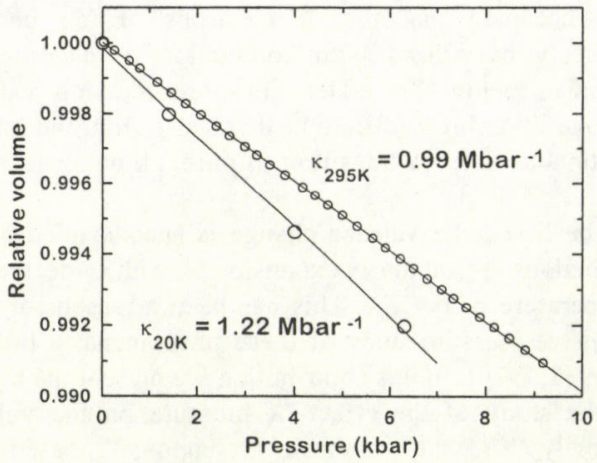

Fig. 4. Relative volume $V(p) / V(0)$ vs. applied pressure $p$ at temperatures $T=295 \mathrm{~K}$ and $T=20 \mathrm{~K}$

We have observed significantly higher value of the compressibility $\kappa$ at temperature $20 \mathrm{~K}$ below $T_{V}\left(\kappa_{l l}=\right.$ $\left.1.22 \mathrm{Mbar}^{-1}\right)$ than above $T_{V}$ at $295 \mathrm{~K} \quad\left(\kappa_{l l I}=0.99\right.$ $\mathrm{Mbar}^{-1}$ ) and the $\lambda$-shaped giant increase of the compressibility near $T_{V}$ ( see Fig. 5). 


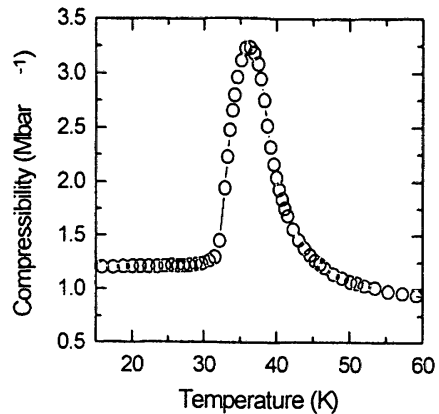

Fig. 5. Temperature dependence of compressibility in the vicinity of the transition temperature $T_{V}$

\section{Discussion and Conclusions}

Recently, the thermal expansion of the intermediate valence rare-earth compounds, with low temperature deviations, has been theoretically predicted and qualitatively described [11,12]. A well-documented phenomenological effect, that an increasing occupation of the rare earth $f$-shell, $n_{f}$, leads to a radial expansion of the valence orbitals (and also to a corresponding expansion of the crystal lattice) due to the enhanced screening of the nuclear charges, has been taken into account. The size of this effect is expressed by the known relation $d \ln V / d n_{f} \approx 0.15$ [11].

Assuming that the volume anomaly in $\mathrm{YbInCu}_{4}, \omega_{v}=$ $3 \Delta L / L=0.43 \%$ at $T_{V}$, is caused by the change of the $\mathrm{Yb}$ atomic volume only, we have to use metallic radii of the relevant atoms to recalculate this anomaly with respect to the $\mathrm{Yb}$ atoms. We have estimated that the volume increase per $\mathrm{Yb}$ atomic volume in the $\mathrm{YbInCu}_{4}$ compound is $\approx 1.3 \%$ and the parameter $d \ln V_{Y b} d d n_{f}=$ $d \ln V_{Y b} / d v \approx 0.13\left(\approx 0.16\right.$ in the case of $\mathrm{YbAl}_{2}$, using the same procedure). This result surprisingly offers a possibility to describe phenomenologically the volume anomaly at the first-order valence change in $\mathrm{YbInCu}_{4}$ as a consequence of the increasing screening of the charge of $\mathrm{Yb}$ nucleus. On the other hand, the smooth decrease of the volume anomaly under pressure shows the continuous increase of the $\mathrm{Yb}$ valence and the decreasing abrupt valence change $\Delta v$ at $T_{V}$ with increasing pressure.

The pressure dependence of valence $v$ in pure $\mathrm{Yb}$

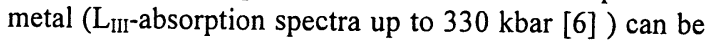
fitted by expression :

$$
v=2+0.005^{*} p-0.000007^{*} p^{2}
$$

where $p$ is pressure in kbar. A rate of the continuous increase of the $\mathrm{Yb}$ valence in the $\mathrm{YbInCu}_{4}$ compound under pressure is well comparable with the initial rate of the continuous pressure increase of the valence in the pure $\mathrm{Yb}$. This fact is surprising, having in mind a significant difference in the $\mathrm{Yb}$-valence in the $\mathrm{Yb}$ and the $\mathrm{YbInCu}_{4}$ compound under normal pressure.

The $\lambda$-shaped giant increase of the compressibility $\kappa$ near $T_{V} \quad$ (see Fig.5) is compatible with a peak on the temperature dependence of specific heat $c_{p}$ at $T_{V}$ [1]. The independent measurements of the isothermal compressibility of the $\mathrm{YbInCu}_{4}$ compound, $\kappa_{\mathrm{II}}$ below and $\kappa_{\text {III }}$ above $T_{V}$, have shown that both values well satisfy a simple relation:

$$
\kappa_{\text {II }}=\kappa_{\text {III }}+d \omega_{v} / d p
$$

$\left(d \omega_{\nu} / d p=0.21 \mathrm{Mbar}^{-1}\right)$. The compressibility $\mathrm{\kappa}_{\mathrm{III}}$ reflects mainly compressibility of Indium and Copper, $\mathrm{K}\left(\mathrm{InCu}_{4}\right)$ $\sim 1.06 \mathrm{Mbar}^{-1}$ while $\mathrm{\kappa}\left(\mathrm{Yb}^{\text {metal }}\right)=7.52 \mathrm{Mbar}^{-1}$. The high value of compressibility $\kappa_{\text {II }}$ below $T_{V}$ seems to be caused only by the pressure dependence of the volume anomaly that accompanies the first-order $\mathrm{Yb}$-valence change in the $\mathrm{YbInCu}_{4}$ compound. This observation supports the conclusion that the enhanced screening of the nuclear charges due to the increasing occupation of $f$-shell (without significant change of cohesive interatomic interactions) is the origin of the corresponding expansion of the crystal lattice.

\section{Acknowledgment}

We acknowledge financial support from Project No. 202/96/0207 GA CR and No. 1010622 GA AS CR.

\section{References}

[1] I.Felner et al., Phys.Rev. B 35, 6956 (1987).

[2] I.Nowik, I.Felner, J.Voiron, J.Beille, A.Najib, E. duTremolet deLacheisserie, and G.Gratz, Phys. Rev. B 37, 5633 (1988).

[3] K.Kojima, H.Hayashi, A.Minami, Y.Kasamatsu, and T.Hihara, J.Magn.Mag.Mats. 81, 267 (1989).

[4] K.Kojima, Y.Nakai, T.Suzuki, H.Asano, F.Izumi, T.Fujita, and T. Hihara, J.Phys.Soc.Japan 59, 792 (1990).

[5] H.Nakamura, K.Nakajima, Y.Kitaoka, K.Asayama, K.Yoshimura, and T.Nitta, J.Phys.Soc.Japan 59, 28 (1990).

[6] K.Syassen, G.Wortmann, J.Feldhaus, K.H.Frank, and G.Kaindl, Phys.Rev. B 26, 4745 (1982).

[7] R.G.Graham, J.S.Lord, P.Riedi, Y.Yamada, H.Nakamura, and K.Yoshimura, J.Magn.Mag.Mats.104-107, 641 (1992).

[8] T.Matsumoto, T.Shimizu, Y.Yamada, and K.Yoshimura, J.Magn.Mag.Mats.104-107, 647 (1992).

[9] K.Kojima, K.Hiraoka, H.Takahashi, N.Mori and T.Hihara, J.Magn.Mag.Mats.140-144, 1241 (1995).

[10] J.M.De Teresa, Z.Arnold, A.delMoral, M.R.Ibarra, D.T. Adroja, and B.Rainford, Solid State Commun. 99, 911 (1996).

[11] E.Müller-Hartmann, Solid State Commun. 31, 113 (1979).

[12] T.Venkatappa Rao, G.Reddy, and A.Ramakanth, Solid State Commun. 87, 157 (1993). 